\title{
The workload of the Interventional Cardiology Centers from Romania: a 2019 overview and 20I4-20I9 trends
}

\author{
Lucian Predescu', Marin Postu'
}

\begin{abstract}
This article provides data of the workload of the interventional cardiology centers from Romania during 2019. Members from all interventional cardiology centers from Romania were requested to fill a standard form about the total number of various procedures performed during 2019. The report highlights the total number of coronary interventions, peripheral interventions, and interventions for structural heart diseases, that were performed in 2019 in Romania. A comparison of the workload of the interventional cardiology centers from Romania between 2014 to 2019 was done.
\end{abstract}

Keywords: interventional cardiology, percutaneous coronary interventions, stents.

Rezumat: Acest articol oferă date despre volumul de lucru al centrelor de cardiologie intervențională din România în cursul anului 2019. Membrii din toate centrele de cardiologie intervențională din România au fost rugați să completeze un formular standard despre numărul total de proceduri intervențioale efectuate în cursul anului 2019. Raportul evidențiază numărul total de intervenții coronariene, intervenții periferice și intervenții pentru boli structurale cardiace, care au fost efectuate în 2019 în România. S-a făcut o comparație între volumul de lucru al centrelor de cardiologie intervențională din România între anii 2014 și 2019.

Cuvinte cheie: cardiologie intervențională, intervenții coronariene percutane, stenturi.

This article provides data of the workload of the interventional cardiology centers from Romania during 2019 and it highlights the trends in coronary or peripheral interventions between 2014 and $2019^{1-5}$. Members from all interventional cardiology centers from Romania were requested to fill a standard form about the total number of various procedures performed during 2019. Data was collected from 34 of 36 interventional cardiology centers from Romania at the initiative of the Interventional Cardiology Working Group of Romanian Society of Cardiology.

Table I summarizes the data from 2019 provided by 34 public and private interventional cardiology centers from Romania. Currently, there are 36 interventional cardiology centers in Romania, of which 12 are private centers and 24 public centers. In 2019, in Romania were 58 interventional cardiologists in training.

\footnotetext{
' „Prof. Dr. C.C. Iliescu” Emergency Institute for Cardiovascular Diseases, Bucharest, Romania
}

The annual number of PCls in every interventional cardiology center from Romania is represented in Figure I. A direct comparison of data from 2014 to 2018 was done, that highlights the continuous increase of workload of the interventional cardiology centers from Romania (Figure 2). However, we can see that the sharp increase in the total number of PCls between 2014-2016 flattened between 2017-2019. In 2019, in Romania, $21783 \mathrm{PCls}$ were performed.

In 2019 the downward trend of the total number of primary PCls performed in STEMI patients continues (Figure 3). This can reveal problems in funding the program or how these cases are reported.

From 2014 to 2019 the total number of implanted stents and the percentage of drug eluting stents increased yearly (Figure 4). In 2019 were implanted 30207 drug eluting stents.

\section{Contact address:}

Marin Postu

„Prof. Dr. C.C. Iliescu” Emergency Institute for Cardiovascular

Diseases, Bucharest, Romania.

E-mail:marin_postu@yahoo.com 
Although, from 2014 to 2018 the total number of peripheral angioplasties doubled, in 2019 there was a decreased in this regard by $13 \%$ (Figure 5 ).

Although the number of TAVI procedures doubled every year, the number of endovascular aortic repair procedures and interventions in structural heart diseases declined in 2019 compared to 2018 (Figure 6).
There is a continuous need of implementing a Mandatory Interventional Cardiology National Registry, capable of monitoring objectively these key health care services in a standard manner. This national registry can guide further development of the interventional cardiology in Romanial-5.

Table I. Reported data from all Interventional Cardiology Centers from Romania in 2019

\begin{tabular}{|c|c|c|c|c|c|c|c|}
\hline City & Bucuresti & Bucuresti & Bucuresti & Bucuresti & Bucuresti & Bucuresti & Bucuresti \\
\hline Hospital & $\begin{array}{c}\text { IUBCV } \\
\text { CC Iliescu }\end{array}$ & SUUB & Floreasca & \begin{tabular}{c|} 
Sp. \\
Agrippa \\
Ionescu \\
\end{tabular} & $\begin{array}{c}\text { Sp. } \\
\text { Militar }\end{array}$ & $\begin{array}{c}\text { Sp. } \\
\text { Bagdasar } \\
\text { Arseni } \\
\end{array}$ & Sp. Elias \\
\hline Nr. Of Cathlabs & 2 & 2 & 1 & 1 & 2 & 1 & 2 \\
\hline Nr. Independent operators & 7 & 6 & 5 & 3 & 4 & 2 & 6 \\
\hline $\mathrm{Nr}$. of in training operators & 12 & 8 & 3 & 0 & 3 & 2 & 4 \\
\hline \multicolumn{8}{|l|}{ Coronary Interventions } \\
\hline Coronarography & 4233 & 1070 & 2340 & 1290 & 2226 & 821 & 532 \\
\hline Total nr. of PCI & 1725 & 1272 & 1305 & 256 & 883 & 411 & 761 \\
\hline PCI without stents & 134 & 70 & 15 & 2 & 12 & 4 & 40 \\
\hline PCI with stents & 1591 & 1202 & 1290 & 254 & 871 & 407 & 721 \\
\hline STEMI & 687 & 1116 & 910 & 12 & 222 & 43 & 294 \\
\hline PCI left main & 89 & 65 & NR & NR & 23 & 32 & NR \\
\hline Stents per patient & 1.52 & 1.46 & 1.38 & 1.35 & 1.34 & 1.1 & 1.1 \\
\hline Total nr. of stents & 2428 & 1863 & 1784 & 345 & 1190 & 442 & 853 \\
\hline Nr. of DES & 2428 & 1805 & 1784 & 345 & 1093 & 438 & 846 \\
\hline DES/stents ratio $(\%)$ & 100 & 97 & 100 & 100 & 92 & 99 & 99 \\
\hline BVS & 0 & 0 & 0 & 0 & 3 & 0 & 0 \\
\hline Cutting, Scoring & 9 & 6 & 3 & 3 & 16 & 1 & 0 \\
\hline Rotabalation & 9 & 0 & 10 & 0 & 0 & 0 & 0 \\
\hline FFR/IFR & 11 & 10 & 20 & 18 & 0 & 0 & 0 \\
\hline IVUS & 13 & 3 & 12 & 1 & 0 & 0 & 0 \\
\hline OCT & 0 & 8 & 9 & 1 & 0 & 0 & 0 \\
\hline \multicolumn{8}{|l|}{ Peripheral interventions } \\
\hline Peripheral PTA & 265 & NR & 0 & 35 & 232 & 2 & 14 \\
\hline Renal PTA & 6 & 25 & 0 & 1 & 13 & 1 & 0 \\
\hline Carotid PTA & 17 & 67 & 0 & 25 & 25 & 0 & 0 \\
\hline $\begin{array}{l}\text { Total nr. of stents in } \\
\text { peripheral PTA }\end{array}$ & 195 & NR & 0 & 37 & 191 & 3 & 4 \\
\hline Peripheral angiography & 755 & NR & 0 & 321 & 510 & 84 & 100 \\
\hline \multicolumn{8}{|l|}{ Other interventions } \\
\hline TAVI & 133 & 31 & 0 & 16 & 11 & 0 & 0 \\
\hline MitraClip & 0 & 0 & $\overline{0}$ & 0 & 0 & 0 & 0 \\
\hline Aortic endoprothesis & 12 & 1 & 0 & 4 & 3 & 0 & 0 \\
\hline $\begin{array}{l}\text { Interventions in structural } \\
\text { heart disease }\end{array}$ & 22 & 8 & 0 & 0 & 0 & 0 & 0 \\
\hline
\end{tabular}

* Number of peripheral angioplasty (lower and upper limbs artery, not including carotid arteries)

** Interventions in cardiac structural diseases: percutaneous closures of DSA, DSV, PFO, arterial duct, balloon valvulotomies, left atrial appendage closure

(PCI - percutaneous coronary intervention, STEMI - ST segment elevation myocardial infarction, DES - drug eluting stent, BVS - bioresorbable vascular scaffold, FFR - fractional flow reserve, IFR - instantaneous wave-free ratio, IVUS - intravascular ultrasound, OCT - optical coherence tomography, PTA - percutaneous transluminal angioplasty, TAVI - transcatheter aortic valve implantation). 


\begin{tabular}{|c|c|c|c|c|c|c|c|}
\hline City & Bucuresti & Bucuresti & Bucuresti & Bucuresti & Bucuresti & Bucuresti & $\begin{array}{c}\text { Cluj } \\
\text { Napoca }\end{array}$ \\
\hline Hospital & $\begin{array}{l}\text { Sp. Sf. } \\
\text { Ioan }\end{array}$ & Sanador & $\begin{array}{c}\text { Ares - } \\
\text { Monza } \\
\text { Bucuresti }\end{array}$ & Colentina & Medlife & $\begin{array}{c}\text { CDT } \\
\text { Victor } \\
\text { Babes } \\
\end{array}$ & $\begin{array}{c}\text { Institutul } \\
\text { Inimii } \\
\text { Cluj }\end{array}$ \\
\hline Nr. Of Cathlabs & 1 & 2 & 4 & 1 & 1 & 1 & 2 \\
\hline Nr. Independent operators & 1 & 10 & 7 & 1 & 3 & 2 & 10 \\
\hline Nr. of in training operators & 0 & 0 & 1 & 1 & 0 & 0 & 1 \\
\hline \multicolumn{8}{|l|}{ Coronary Interventions } \\
\hline Coronarography & 403 & 1117 & 1358 & 318 & 517 & 67 & 2035 \\
\hline Total nr. of PCI & 104 & 132 & 423 & 100 & 161 & 32 & 1713 \\
\hline PCI without stents & 7 & 3 & 15 & 0 & 5 & 2 & 273 \\
\hline PCI with stents & 97 & 129 & 408 & 100 & 156 & 30 & 1440 \\
\hline STEMI & 7 & 9 & 10 & 1 & 4 & 0 & 418 \\
\hline PCI left main & 2 & 3 & 70 & 6 & 10 & 0 & 57 \\
\hline Stents per patient & 1.28 & 1 & 2.5 & 1.6 & 1.4 & 2.36 & 1.47 \\
\hline Total nr. of stents & 134 & 129 & 1060 & 159 & 221 & 71 & 2529 \\
\hline Nr. of DES & 125 & 129 & 1060 & 159 & 221 & 71 & 2500 \\
\hline DES/stents ratio $(\%)$ & 93 & 100 & 100 & 100 & 100 & 100 & 98 \\
\hline BVS & 0 & 0 & 0 & 0 & 0 & 0 & 0 \\
\hline Cutting, Scoring & 0 & 0 & 21 & 2 & 5 & 1 & 20 \\
\hline Rotabalation & 0 & 3 & 9 & 0 & 0 & 0 & 3 \\
\hline FFR/IFR & 0 & 0 & 0 & 0 & 0 & 0 & 102 \\
\hline IVUS & 0 & 0 & 2 & 0 & 0 & 0 & 68 \\
\hline OCT & 0 & 0 & 0 & 0 & 0 & 0 & 8 \\
\hline \multicolumn{8}{|l|}{ Peripheral interventions } \\
\hline Peripheral PTA & 21 & 60 & 68 & 8 & 37 & 4 & 94 \\
\hline Renal PTA & 7 & 2 & 2 & 0 & 1 & 0 & 11 \\
\hline Carotid PTA & 0 & 1 & 15 & 0 & 12 & 0 & 32 \\
\hline $\begin{array}{l}\text { Total nr. of stents in } \\
\text { peripheral PTA }\end{array}$ & 27 & 15 & 36 & 4 & 77 & 4 & 121 \\
\hline Peripheral angiography & 134 & 446 & 315 & 21 & 174 & 13 & 287 \\
\hline \multicolumn{8}{|l|}{ Other interventions } \\
\hline TAVI & 0 & 20 & 37 & 0 & 0 & 0 & 90 \\
\hline MitraClip & 0 & 0 & 3 & 0 & 0 & 0 & 0 \\
\hline Aortic endoprothesis & 0 & 8 & 1 & 0 & 0 & 0 & 21 \\
\hline $\begin{array}{l}\text { Interventions in structural } \\
\text { heart disease }\end{array}$ & 0 & 1 & 27 & 0 & 5 & 0 & 5 \\
\hline
\end{tabular}




\begin{tabular}{|c|c|c|c|c|c|c|c|}
\hline City & $\begin{array}{c}\text { Cluj } \\
\text { Napoca }\end{array}$ & $\begin{array}{c}\text { Cluj } \\
\text { Napoca }\end{array}$ & Cluj Napoca & Iasi & Iasi & $\begin{array}{l}\text { Târgu } \\
\text { Mureș }\end{array}$ & $\begin{array}{l}\text { Târgu } \\
\text { Mureș }\end{array}$ \\
\hline Hospital & $\begin{array}{l}\text { SCJU } \\
\text { Cluj }\end{array}$ & $\begin{array}{l}\text { Monza- } \\
\text { Ares Cluj }\end{array}$ & \begin{tabular}{|c|} 
Sp. \\
Recuperare \\
Cluj
\end{tabular} & IBCV Iaşi & $\begin{array}{c}\text { Arcadia } \\
\text { Iasi }\end{array}$ & $\begin{array}{l}\text { IBCV Tg } \\
\text { Mureș }\end{array}$ & $\begin{array}{l}\text { CardioMed } \\
\text { Tg. Mures }\end{array}$ \\
\hline Nr. Of Cathlabs & 1 & 2 & 1 & 2 & 1 & 2 & 2 \\
\hline Nr. Independent operators & 3 & 4 & 2 & 6 & 2 & 5 & 5 \\
\hline $\mathrm{Nr}$. of in training operators & 3 & 1 & 1 & 2 & 0 & 1 & 0 \\
\hline \multicolumn{8}{|l|}{ Coronary Interventions } \\
\hline Coronarography & 1329 & 342 & 751 & 3500 & 971 & 1924 & 236 \\
\hline Total nr. of PCI & 741 & 166 & 305 & 1456 & 156 & 1278 & 251 \\
\hline PCI without stents & 28 & 8 & 35 & 84 & 4 & 135 & 30 \\
\hline PCI with stents & 713 & 158 & 270 & 956 & 152 & 1143 & 221 \\
\hline STEMI & 192 & 0 & 10 & 756 & 0 & 359 & 0 \\
\hline PCI left main & 52 & NR & 7 & 50 & NR & 195 & NR \\
\hline Stents per patient & 2.12 & 1.6 & 1.4 & 1.4 & 1.24 & 1.29 & 1.21 \\
\hline Total nr. of stents & 1482 & 251 & 322 & 2582 & 194 & 1649 & 290 \\
\hline Nr. of DES & 1482 & 251 & 310 & 2292 & 194 & 1649 & 286 \\
\hline DES/stents ratio (\%) & 1 & 100 & 96 & 88 & 100 & 100 & 98 \\
\hline BVS & 0 & 0 & 0 & 0 & 0 & 0 & 0 \\
\hline Cutting, Scoring & 0 & 7 & 0 & 12 & 0 & 20 & 0 \\
\hline Rotabalation & 0 & 1 & 0 & 0 & 0 & 7 & 0 \\
\hline FFR/IFR & 0 & 5 & 0 & 0 & 0 & 40 & 36 \\
\hline IVUS & 0 & 0 & 0 & 0 & 0 & 0 & 36 \\
\hline $\mathrm{OCT}$ & 14 & 0 & 0 & 0 & 0 & 26 & 37 \\
\hline & & & & & & & \\
\hline \multicolumn{8}{|l|}{ Peripheral interventions } \\
\hline Peripheral PTA & 155 & 64 & 75 & 40 & 28 & 85 & 66 \\
\hline Renal PTA & 4 & 1 & 2 & 5 & 0 & 1 & 0 \\
\hline Carotid PTA & 3 & 22 & 0 & 14 & 11 & 33 & 0 \\
\hline $\begin{array}{l}\text { Total nr. of stents in } \\
\text { peripheral PTA }\end{array}$ & 154 & 81 & 0 & 50 & NR & 59 & 1 \\
\hline Peripheral angiography & 339 & 57 & 152 & 142 & 109 & 250 & 20 \\
\hline \multicolumn{8}{|l|}{ Other interventions } \\
\hline$\overline{\text { TAVI }}$ & 0 & 0 & 0 & 13 & 0 & 29 & 0 \\
\hline MitraClip & 0 & 0 & 0 & 0 & 0 & 0 & 0 \\
\hline Aortic endoprothesis & 0 & 1 & 0 & 7 & 0 & 21 & 0 \\
\hline $\begin{array}{l}\text { Interventions in structural } \\
\text { heart disease }\end{array}$ & 0 & 9 & 0 & 7 & 3 & 41 & 0 \\
\hline
\end{tabular}




\begin{tabular}{|c|c|c|c|c|c|c|c|}
\hline City & $\begin{array}{l}\text { Târgu } \\
\text { Mureș }\end{array}$ & $\begin{array}{l}\text { Târgu } \\
\text { Mureș }\end{array}$ & Timișoara & Braşov & Braşov & Oradea & Arad \\
\hline Hospital & $\begin{array}{c}\text { SCJU Tg } \\
\text { Mureș }\end{array}$ & $\begin{array}{l}\text { Nova Vita } \\
\text { Tg. Mures }\end{array}$ & $\begin{array}{c}\text { IBCV } \\
\text { Timişoara }\end{array}$ & $\begin{array}{l}\text { Clinicco } \\
\text { Brasov }\end{array}$ & $\begin{array}{l}\text { Sp. Jud. } \\
\text { Brasov }\end{array}$ & $\begin{array}{l}\text { Sp. Jud. } \\
\text { Oradea }\end{array}$ & $\begin{array}{c}\text { Cardiolife } \\
\text { Arad }\end{array}$ \\
\hline Nr. Of Cathlabs & 2 & 1 & 2 & 1 & 2 & 2 & NR \\
\hline Nr. Independent operators & 5 & 6 & 7 & 3 & 3 & 4 & NR \\
\hline Nr. of in training operators & 7 & 0 & 2 & 0 & 1 & 0 & NR \\
\hline \multicolumn{8}{|l|}{ Coronary Interventions } \\
\hline Coronarography & 381 & 1306 & 3774 & 1657 & 1743 & 1703 & NR \\
\hline Total nr. of PCI & 682 & 97 & 1907 & 432 & 997 & 895 & NR \\
\hline PCI without stents & 66 & 0 & 87 & 10 & 54 & 3 & NR \\
\hline PCI with stents & 616 & 97 & 1820 & 422 & 943 & 892 & NR \\
\hline STEMI & 453 & 0 & 870 & 8 & 465 & 502 & NR \\
\hline PCI left main & NR & 2 & 65 & NR & 50 & 115 & NR \\
\hline Stents per patient & 1.39 & 1.25 & 1.74 & 1.36 & 1.3 & 2 & NR \\
\hline Total nr. of stents & 861 & 122 & 3330 & 590 & 1250 & 1794 & NR \\
\hline Nr. of DES & 403 & 122 & 2631 & 585 & 1250 & 1675 & NR \\
\hline DES/stents ratio (\%) & 46 & 100 & 79 & 99 & 100 & 93 & NR \\
\hline BVS & 0 & 0 & 0 & 0 & 0 & 0 & NR \\
\hline Cutting, Scoring & 0 & 0 & 2 & 2 & 25 & 0 & NR \\
\hline Rotabalation & 0 & 0 & 0 & 8 & 0 & 4 & NR \\
\hline FFR/IFR & 2 & 0 & 16 & 0 & 49 & 8 & NR \\
\hline IVUS & 0 & 0 & 0 & 0 & 0 & 0 & NR \\
\hline $\mathrm{OCT}$ & 13 & 0 & 2 & 0 & 20 & 0 & $\mathrm{NR}$ \\
\hline \multicolumn{8}{|l|}{ Peripheral interventions } \\
\hline Peripheral PTA & 14 & 18 & 59 & 16 & 2 & 0 & NR \\
\hline Renal PTA & 0 & 0 & 10 & 0 & 0 & 0 & $\mathrm{NR}$ \\
\hline Carotid PTA & 0 & 4 & 75 & 3 & 20 & 0 & NR \\
\hline $\begin{array}{l}\text { Total nr. of stents in } \\
\text { peripheral PTA }\end{array}$ & 1 & 8 & 146 & 18 & 2 & 0 & NR \\
\hline Peripheral angiography & 80 & 80 & 1253 & 3 & 3 & 98 & NR \\
\hline \multicolumn{8}{|l|}{ Other interventions } \\
\hline TAVI & 2 & 0 & 19 & 1 & 0 & 0 & NR \\
\hline MitraClip & 0 & 0 & 0 & 0 & 0 & 0 & NR \\
\hline Aortic endoprothesis & 0 & 0 & 4 & 3 & 0 & 0 & $\mathrm{NR}$ \\
\hline $\begin{array}{l}\text { Interventions in structural } \\
\text { heart disease }\end{array}$ & 0 & 0 & 4 & 2 & 0 & 0 & NR \\
\hline
\end{tabular}




\begin{tabular}{|c|c|c|c|c|c|c|c|c|}
\hline City & Suceava & Baia Mare & Sibiu & Sibiu & Constanta & Ploiesti & Pitesti & Craiova \\
\hline Hospital & $\begin{array}{l}\text { Sp. Jud. } \\
\text { Suceava }\end{array}$ & $\begin{array}{c}\text { Sp. Jud. } \\
\text { Baia Mare }\end{array}$ & $\begin{array}{c}\text { Polisano- } \\
\text { Medlife }\end{array}$ & $\begin{array}{c}\text { Sp. } \\
\text { Jud. } \\
\text { Sibiu }\end{array}$ & \begin{tabular}{|c|} 
Monza - \\
Ares \\
Constanta
\end{tabular} & $\begin{array}{l}\text { Sp. Jud. } \\
\text { Ploiesti }\end{array}$ & $\begin{array}{l}\text { Sp. Jud. } \\
\text { Pitesti }\end{array}$ & $\begin{array}{l}\text { Sp. Jud. } \\
\text { Craiova }\end{array}$ \\
\hline Nr. Of Cathlabs & $\mathrm{NR}$ & 2 & 1 & 1 & 1 & 2 & 1 & 1 \\
\hline Nr. Independent operators & $\mathrm{NR}$ & 2 & 1 & 2 & 1 & 2 & 1 & 1 \\
\hline Nr. of in training operators & $\mathrm{NR}$ & 1 & 0 & 1 & 0 & 0 & 1 & 2 \\
\hline \multicolumn{9}{|l|}{ Coronary Interventions } \\
\hline Coronarography & NR & 252 & 995 & 586 & 1375 & 737 & 558 & 717 \\
\hline Total nr. of PCI & NR & 679 & 423 & 396 & 690 & 374 & 427 & 153 \\
\hline PCI without stents & NR & 64 & 17 & 10 & 36 & 26 & 26 & 1 \\
\hline PCI with stents & NR & 615 & 406 & 340 & 654 & 348 & 401 & 152 \\
\hline STEMI & NR & 243 & 40 & 32 & 332 & 184 & 78 & 24 \\
\hline PCI left main & NR & 27 & 14 & 12 & 5 & 5 & NR & 5 \\
\hline Stents per patient & NR & 1.46 & 1.6 & 1.72 & 1.3 & 1.1 & 1.1 & 1.61 \\
\hline Total nr. of stents & NR & 895 & 678 & 586 & 850 & 416 & 459 & 246 \\
\hline Nr. of DES & NR & 877 & 676 & 586 & 850 & 400 & 447 & 237 \\
\hline DES/stents ratio (\%) & NR & 98 & 99 & 100 & 100 & 96 & 97 & 96 \\
\hline BVS & NR & 0 & 0 & $\overline{0}$ & 0 & $\overline{0}$ & 0 & 0 \\
\hline Cutting, Scoring & NR & 10 & 20 & 0 & 0 & 0 & 11 & 0 \\
\hline Rotabalation & NR & 0 & 1 & 0 & 0 & 0 & 3 & 0 \\
\hline FFR/IFR & $\mathrm{NR}$ & 22 & 10 & 0 & 0 & 0 & 11 & 0 \\
\hline IVUS & NR & 0 & 0 & 0 & 0 & 0 & 10 & 0 \\
\hline OCT & NR & 8 & 0 & 2 & 0 & 0 & 0 & 0 \\
\hline \multicolumn{9}{|l|}{ Peripheral interventions } \\
\hline Peripheral PTA & NR & 17 & 16 & 153 & 0 & 8 & 140 & 0 \\
\hline Renal PTA & $\overline{N R}$ & 0 & 1 & 0 & $\overline{0}$ & $\overline{0}$ & 0 & $\overline{0}$ \\
\hline Carotid PTA & NR & 0 & 0 & 0 & 2 & 0 & 1 & 0 \\
\hline $\begin{array}{l}\text { Total nr. of stents in } \\
\text { peripheral PTA }\end{array}$ & NR & 5 & 11 & 80 & 0 & 1 & 73 & 0 \\
\hline Peripheral angiography & $\mathrm{NR}$ & 119 & 28 & 205 & 0 & 158 & 222 & 37 \\
\hline \multicolumn{9}{|l|}{ Other interventions } \\
\hline TAVI & NR & 0 & 26 & 0 & 0 & 0 & 0 & 0 \\
\hline MitraClip & NR & 0 & 0 & 0 & 0 & 0 & $\overline{0}$ & 0 \\
\hline Aortic endoprothesis & NR & 3 & 24 & 0 & 0 & 0 & 0 & 0 \\
\hline $\begin{array}{l}\text { Interventions in structural } \\
\text { heart disease }\end{array}$ & NR & 0 & 6 & 0 & 0 & 0 & 0 & 0 \\
\hline
\end{tabular}




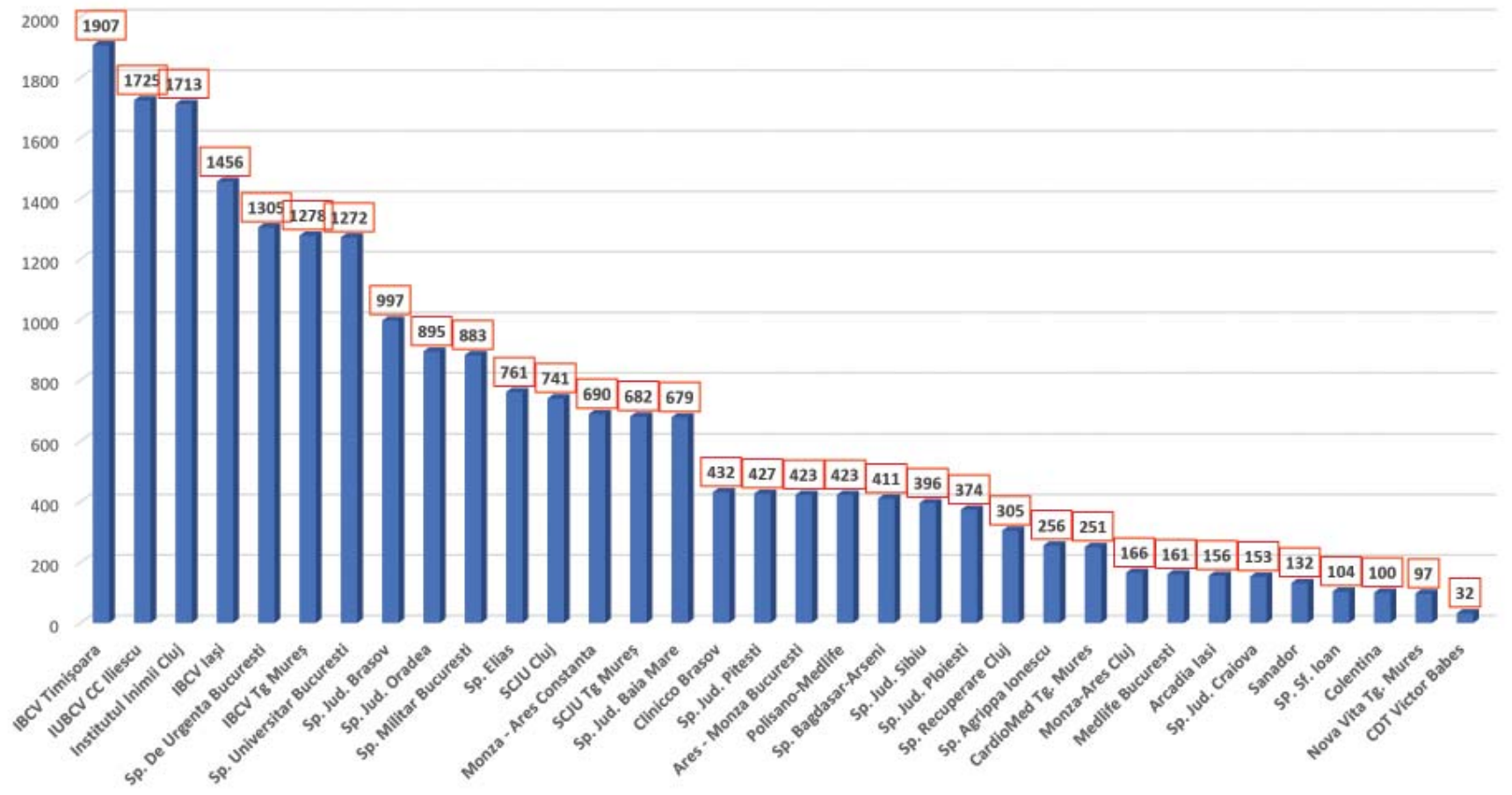

Figure I. Total number of percutaneous coronary interventions in 2019 in interventional centers from Romania.

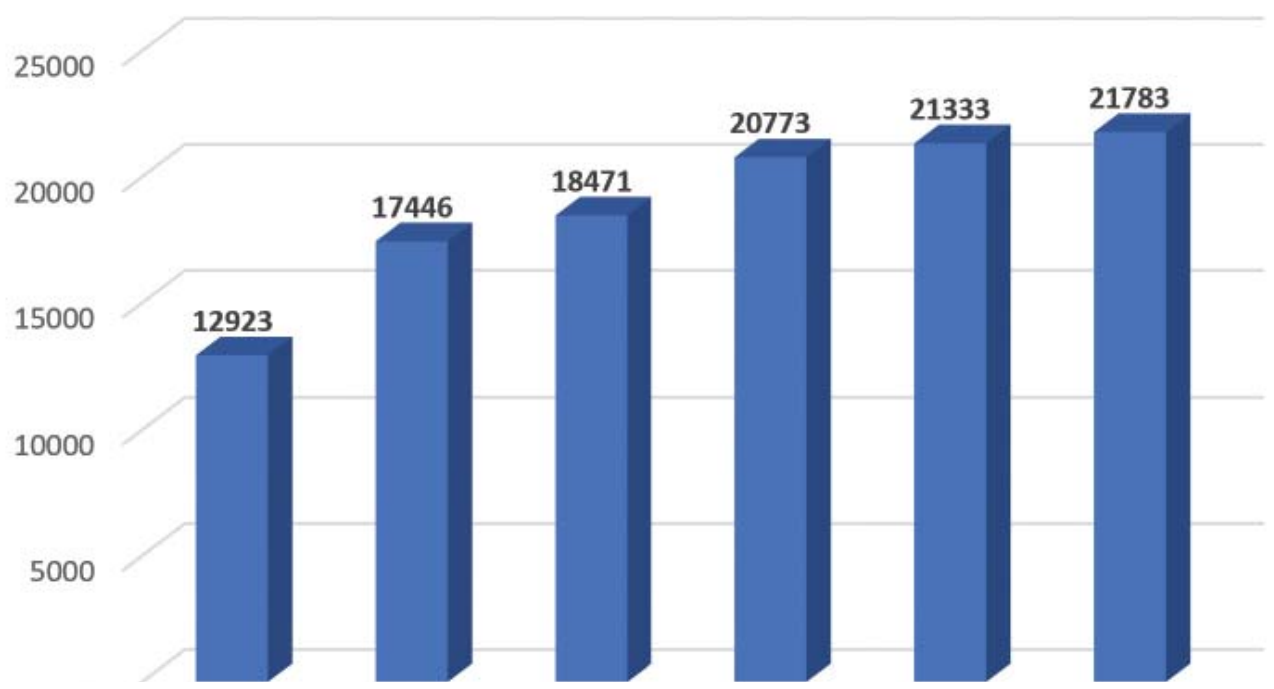

Figure 2. Total number of percutaneous coronary interventions from 2014 to 2019 in Romania. 


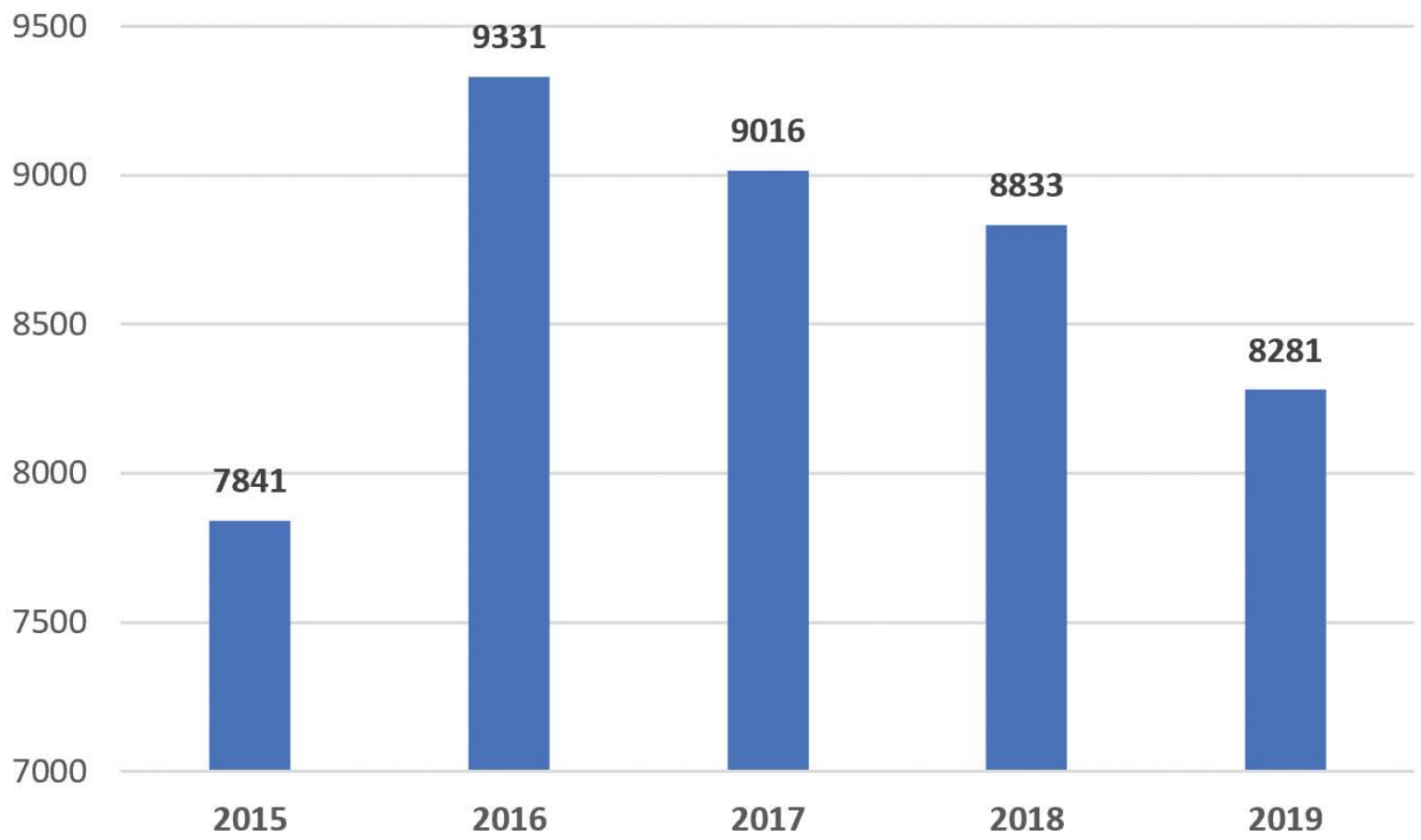

Figure 3. Total number of primary percutaneous coronary interventions in STEMI patients from 2015 to 2019 in interventional centers from Romania (STEMI - ST segment elevation myocardial infarction).

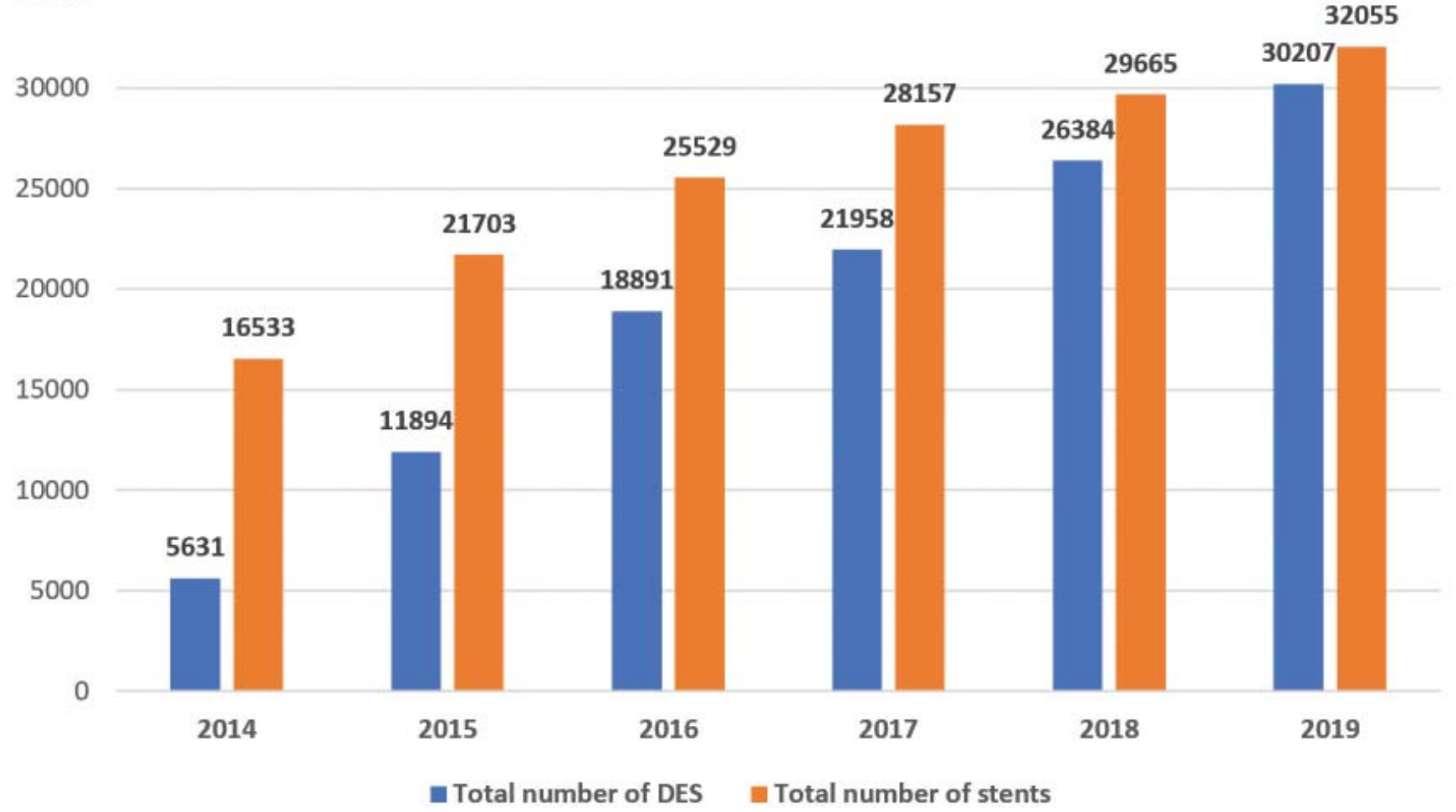

Figure 4. Total number of stents implanted from 2014 to 2019 in Romania (DES - drug eluting stent). 


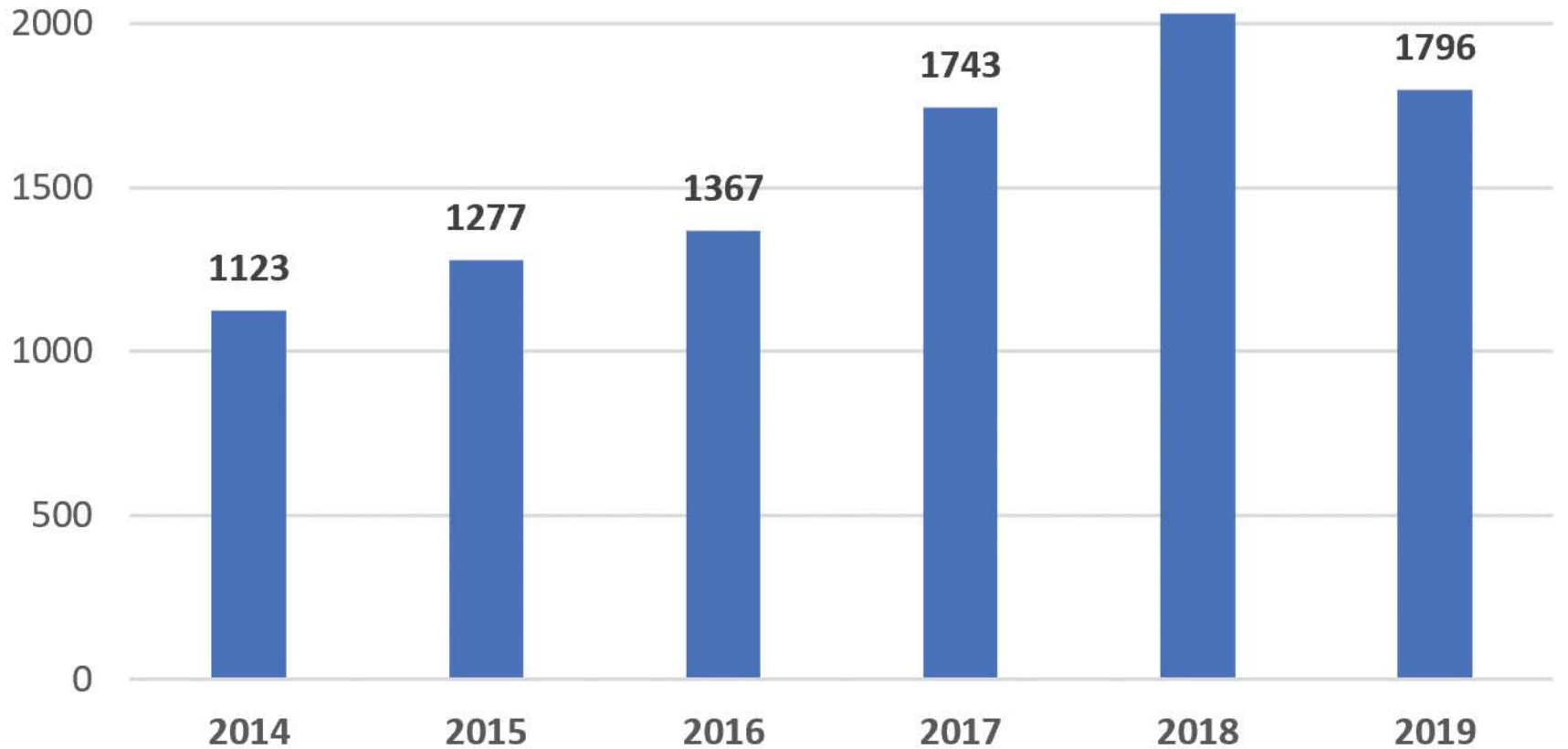

Figure 5. Total number of peripheral angioplasties from 2014 to 2019 in Romania (only in interventional cardiology centers).

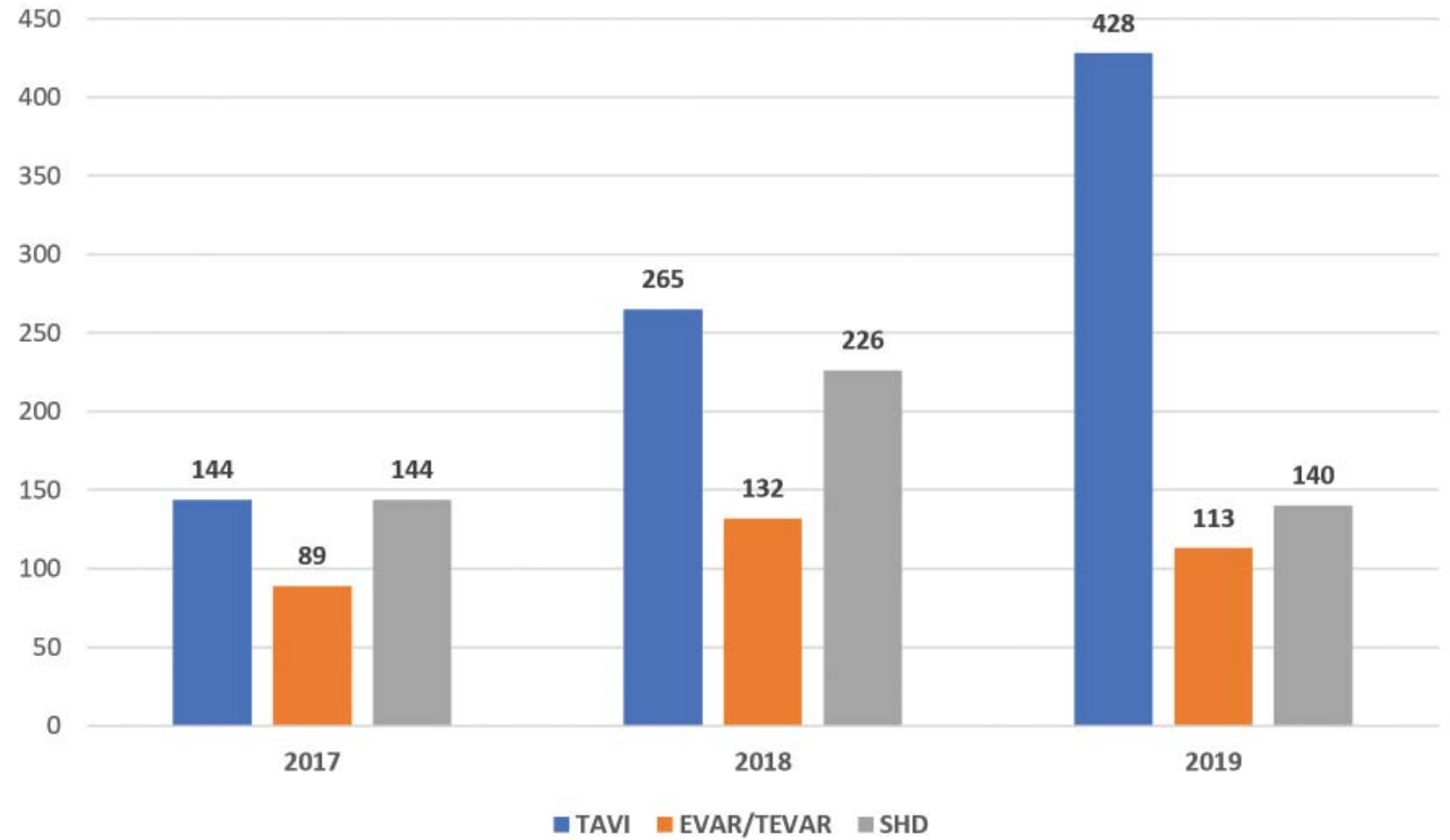

Figure 6. Total number of TAVI, EVAR/TEVAR and SHD interventions performed from 2017 to 2019 in Romania (TAVI - transcatheter aortic valve implantation, EVAR - Endovascular Aneurysm Repair, TEVAR - Thoracic endovascular aortic repair, SHD - structural heart diseases). 
Conflict of interest: none declared.

Acknowledgement: We want to thank for their important contribution to data collection to: Dr. Adrian Bucsa and Prof. Dr. Vlad Iliescu, „Prof. Dr. CC Iliescu" Emergency Institute for Cardiovascular Diseases, Bucharest; Dr. Cristian Udroiu and Prof. Dr. Dragos Vinereanu, Emergency University Hospital, Bucharest; Dr. Lucian Calmac, Emergency Clinic Hospital, Bucharest; Dr. Emanuel Radu, Emergency Clinical Hospital „Prof. Dr. Agrippa lonescu „, Bucharest; Dr. Sebastian Botezatu and Dr. Ruxandra Tudoran, Central Military Emergency University Hospital ,Dr. Carol Davila”, Bucharest; Dr. Valentin Chioncel, ,Bagdasar-Arseni” Clinical Emergency Hospital, Bucharest; Dr. Mihai Melnic, Elias Emergency University Hospital, Bucharest; Dr. Octavian Zara, ,Sf. Ioan” Clinical Emergency Hospital, Bucharest; Dr. Cristina Spinu, Sanador Clinical Hospital, Bucharest; Dr. Oren lancovici, ARES Centers of Excellence in Cardiology and Radiology, Bucharest and Ares-Monza, Constanta and Cluj Napoca; Prof. Dr. Gheorghe Dan and Dr. Emilian Mihai, Colentina Clinical Hospital, Bucharest; Dr. Florina Pinte, Medlife, Bucharest; Dr. Lucian Zarma, „Victor Babes” Private Medical Clinic, Bucharest; Dr. Stefan Mot, Heart Institute „Niculae Stancioiu”, Cluj-Napoca; Prof. Dr. Dan Olinic, County Emergency Hospital, Cluj-Napoca; Dr. Bogdan Dutu, Clinical Recovery Hospital, Cluj-Napoca; Dr. Alexandru Burlacu, Institute of Cardiovascular Diseases „Prof. Dr. George IM Georgescu”, Dr. Igor Nedelciuc, Arcadia, lasi; Prof. Dr. Imre Benedek and Prof. Dr. Teodora Benedek, County Emergency
Hospital and CardioMed, Targu Mures, Dr. Ayman Elkahlout and Dr. Viorel Nicolae, Emergency Institute For Cardiovascular Diseases, Targu Mures; Conf. Dr. loan Tilea, Nova-Vita, Targu Mures; Conf. Dr. Cristian Mornos and Dr. Milovan Slovenski, Institute Of Cardiovascular Disease, Timisoara; Dr. Mihai Ursu, Clinicco, Brasov; Dr. Florin Ortan and Dr. Cristina Popica, County Emergency Hospital, Brasov; Dr. Virgil Popa and Dr. Marius Rus, County Emergency Clinical Hospital, Oradea; Dr. Dan Filip, County Emergency Hospital "Dr. Constantin Opris", Baia Mare; Dr. Nicolae Florescu, Polisano, Sibiu; Dr. Radu Pretorian and Dr. Ioan Bitea, County Emergency Hospital, Sibiu; Dr. Marian Albu and Dr. Victor lorga, County Emergency Hospital, Ploiesti; Dr. Elvis Botu and Dr. Gabriel Stanica, County Emergency Hospital, Pitesti; Dr. Radu Stavaru and Dr. Paul Trasca; County Emergency Hospital, Craiova.

\section{References}

I. Lucian Predescu, Marin Postu, The workload of the Interventional Cardiology Centers from Romania: a 2018 overview and 2014-2018 trends, Romanian Journal of Cardiology, Vol. 29, No. 3, 2019, pag. 4|5-42|

2. Predescu L, Postu M, The activity of the Interventional Cardiology Centers in Romania: a 2017 overview and 2014-2017 trends, Romanian Journal of Cardiology, Vol. 28, No. 2, 2018, pag. I47-I53

3. Postu M, Predescu L, Mot S, The activity of the interventional cardiology centers in Romania: a 2016 overview and 2014-2016 trends, Romanian Journal of Cardiology, Vol. 27, No. 3, 2017, pag. 359-368

4. Postu M, Predescu L, Mot S, The activity of the Interventional Cardiology Centers in Romania: a 2015 overview, Romanian Journal of Cardiology, Vol. 26, No. 3, 2016, pag. 298-309

5. Postu M, Ușurelu C, Predescu L et al., Romanian interventional cardiology centers, 2014-20I5: an overview, Romanian Journal of Cardiology, Vol. 25, No. 2, 20I5, pag. I70-174. 